\title{
Unimodular sequence design under frequency hopping communication compatibility requirements
}

\author{
Peng Ge, Guolong Cui*, Lingjiang Kong and Jianyu Yang
}

\begin{abstract}
The integrated design for both radar and anonymous communication has drawn more attention recently since wireless communication system appeals to enhance security and reliability. Given the frequency hopping (FH) communication system, an effective way to realize integrated design is to meet the spectrum compatibility between these two systems. The paper deals with a unimodular sequence design technique which considers optimizing both the spectrum compatibility and peak sidelobes levels (PSL) of auto-correlation function (ACF). The spectrum compatibility requirement realizes anonymous communication for the $\mathrm{FH}$ system and provides this system lower probability of intercept (LPI) since the spectrum of the FH system is hidden in that of the radar system. The proposed algorithm, named generalized fitting template (GFT) technique, converts the sequence optimization design problem to a iterative fitting process. In this process, the power spectrum density (PSD) and PSL behaviors of the generated sequences fit both PSD and PSL templates progressively. Two templates are established based on the spectrum compatibility requirement and the expected PSL. As noted, in order to ensure the communication security and reliability, spectrum compatibility requirement is given a higher priority to achieve in the GFT algorithm. This algorithm realizes this point by adjusting the weight adaptively between these two terms during the iteration process. The simulation results are analyzed in terms of bit error rate (BER), PSD, PSL, and signal-interference rate (SIR) for both the radar and FH systems. The performance of GFT is compared with SCAN, CAN, FRE, CYC, and MAT algorithms in the above aspects, which shows its good effectiveness.
\end{abstract}

Keywords: Waveform design, Frequency hopping, Anonymous communication, Spectrum compatibility, Auto-correlation function

\section{Introduction}

Spectrum compatibility problem has become a challenging issue for electromagnetic space [1-6]. Although higher quality wireless telecommunication and more accurate remote sensing capabilities require sufficient bandwidth $[7,8]$, the frequency spectrum resource is limited, and special frequency bands with good penetrability [9] or low propagation attenuation are even more fiercely competitive. Hence, the working band of radar has a high opportunity to overlay with that of other electromagnetic radiator systems. Under these circumstances, the spectrum compatibility between radar signals and the

*Correspondence: cuiguolong@uestc.edu.cn

School of Electronic Engineering, University of Electronic Science and

Technology of China, 2006 Xi Yuan Ave, West Hi-tech Zone, Chengdu, China surrounding electromagnetic radiators is essentially necessary. So the problem converts to designing the radar sequence that restrains the electromagnetic energy level over the overlapped spectrum.

Many pieces of work have been devoted to design radar sequences which are capable of spectrum compatibility. For instance, the sequence designing technique proposed by Gerlach [10] can generate sequences with some desired spectral nulls by appending polyphase codes on a stepped frequency modulated pulse. Selesnick and Pillai [11] devote themselves to produce constant modulus waveforms with multiple notches in their frequency spectra (2011). Moreover, some waveform design algorithms are exploited for both spectrum location and auto-correlation function (ACF) requirements. For example, the technique developed by Lindenfeld [12] achieves frequency 
stop-band suppression and range sidelobe minimization by the joint design of the transmit signal and the receive filter (2004). Stop cyclic algorithm new (SCAN), proposed by $\mathrm{He}$ et al. [13], implements the spectrum space operation to consider both the stop-band suppression and the integrated sidelobe level (ISL) suppression (2010). A sparse frequency constant modulus sequence design algorithm, built by Wang and $\mathrm{Lu}$ [14], optimizes both the ISL metric and a penalty function which accounts for the sequence frequency allocation (2011). A spectrumcentric sequence design approach, proposed by Patton et al. [15], minimizes the transmitted energy over some frequency bands under the uni-modularity and ACF masking constraint (2012).

In reality, lots of the actual scenes for target detection are in colored noise or interference environment [16]. Some sequence design techniques have been presented to optimize the signal-to-interference-plus-noiseratio (SINR) in the presence of colored interference. The spectrum of generated sequence is expected to adjust adaptively according to the spectrum shape of colored interference. In this way, spectrum compatibility can be achieved essentially. Li et al. [17] consider the sequence design problem in the presence of colored interference and noise to optimize the SINR for target detection under similarity constraint. The work proposed by De Maio et al. [18] considers the problem of radar sequence design in the presence of colored Gaussian disturbance under similarity constraint. The resolver is based on the semidefinite program (SDP) relaxation and randomization methods.

A joint design of transmit signal and receive filter is proposed by Aubry et al. [19] to consider optimizing the detection performance for a point-like target in the presence of either a homogeneous ground clutter scenario or a heterogeneous mixed land and sea clutter environment under similarity and energy constraints. After that, he and his collaborators considered the similar joint design problem but in a high-reverberating environment in the presence of a homogeneous clutter scenario under a constant modulus constraint [20]. The computational complexity of these two techniques is linear with the number of iterations and polynomial with the receive filter length. Additionally, Aubry et al. considered optimizing the detection performance for a target with unknown Doppler shift and developed a joint design technique to optimize the worst case SINR at the output of the filter bank under both a similarity and an energy constraint on the transmit signal [21].

Furthermore, some pieces of work have considered both radar detection performance and spectrum compatibility requirements. A joint waveform and power spectrum design technique is developed by Turlapaty and Jin [22] to optimize the performance of both the radar and communications systems based on a combined mutual information criterion. Aubry et al. proposed [23] a waveform design technique that deal with the synthesis of optimized radar waveforms ensuring spectral compatibility with the overlayed licensed electromagnetic radiators. Then, he and his collaborators [24] proposed a waveform design technique accounting for SINR and spectrum shape through a suitable modulation of the transmitted waveform energy. After that, Aubry and his other collaborators [25] devised the waveform that opposes a specific control on the interference energy radiated on each shared bandwidth. Both of these two algorithms are based on the method of semi-definite program (SDP) relaxation. Chiriyath et al. [26] proposed a waveform design technique for joint radar SINR enhancement and interference reduction from other telecommunication systems. Last but not the least, a template approximation method, proposed by $\mathrm{Ge}$ et al. [27] can produce unimodular sequences that oppose weighted spectrum compatibility ability and/or weighted regional sidelobe levels of ACF.

Frequency hopping $(\mathrm{FH})$ communication, as one kind of wireless communication modes, plays an important role in the field of military communications for its prominent anti-jamming performance. The low probability of intercept (LPI) feature of the FH system profits from pseudo-random switch-over among multiple channels. While with the great improvements of the spectral analysis implement in the recent 10 years, the frequency locations of FH system channels can be detected. In order to improve the low probability of interception, one suitable way is to make the spectrum of FH channels be hidden in the spectrum of radar radiation on purpose. In this way, it is very difficult for the electronic countermeasure equipment (EW/ECM) to detect and counter.

However, to the best of our knowledge, there have been few pieces of work about the sequence design problem that consider both the radar detection and the $\mathrm{FH}$ communication performance with the spectrum of them coexisting with each other. It is the scope of this paper. The proposed sequence design algorithm considers meeting the bit error rate (BER) requirements from the $\mathrm{FH}$ system with higher priority and optimizing the PSL performance of ACF with a lower priority in the iteration process. The weight in the objective function between these two terms are adaptively adjusted in each iteration to achieve above purpose.

Based on the BER requirement, the required power spectrum density (PSD) of the sequence to generate can be obtained to form a PSD template. The requirements for sidelobe levels of the ACF are also expressed with a ACF template. With a suitable initial sequence, the proposed technique will make the PSD and ACF features of generated sequence fit these two templates in the iteration process. 
The paper is organized as follows. In Section 2, the model for the radar signal model and the problem description of the coexistence scene between radar and the FH system are introduced. In Section 3, the GFT method is proposed as an iterative algorithm. In Section 4, the performance of the proposed algorithm is analyzed in terms of bit error rate (BER), PSD, PSL, and signal-interference rate (SIR) for both radar and FH systems. Finally, Section 5 is devoted to conclusions and proposals for possible future research tracks.

Notation: We adopt the notation of using boldface for vectors $\boldsymbol{a}$ (lower case) and matrices $\boldsymbol{A}$ (uppercase). The $n$th element of $\boldsymbol{a}$ and the $(m, l)$ th entry of $\boldsymbol{A}$ are, respectively, denoted by $a(n)$ and $A(m, l)$. The transpose and the conjugate transpose operators are denoted by the symbols $(\cdot)^{T}$ and $(\cdot)^{\dagger}$, respectively. We use $U(\cdot)$ and $\operatorname{rect}(\cdot)$ to denote union set and rectangular window function, respectively. $\mathbb{R}^{N}$ and $\mathbb{C}^{N}$ are respectively the sets of $N$ dimensional vectors of real and complex numbers. $\mathbb{H}^{N \times N}$ is the sets of $N \times N$ complex matrixs. The Euclidean norm and infinity norm of the vector $\boldsymbol{x}$ are denoted by $\|\boldsymbol{x}\|$ and $\|\boldsymbol{x}\|_{\infty}$, respectively. The letter $j$ represents the imaginary unit (i.e., $j=\sqrt{-1}$ ). For any complex number $x$, we use $\operatorname{Re}(x)$ and $\operatorname{Im}(x)$ to denote, respectively, the real and the imaginary part of $x \cdot \min (\boldsymbol{a})$ denotes the minimum value of all elements in $\boldsymbol{a} .|x|$ and $\angle(\cdot)$ represents the modulus and the phase angle (in radians) of $x$, respectively. Besides, $\mathbb{E}[\cdot]$ denotes the statistical expectation.

\section{System model and problem formulation}

In this section, both the radar system model and the FH communication system model are introduced to formulate the problem.

Consider a radar system, where the baseband equivalent of the transmit pulse is defined by $c(t)$. It is composed of $N$ linearly modulated sub-pulses; the sequence element for the $n$th sub-pulse is denoted by $c(n) \in \mathbb{C}$ with $|c(n)|=1$, for $(0 \leq n \leq N-1)$. Therefore, $c(t)$ can be given as

$$
c(t)=\sum_{n=0}^{N-1} c(n) \operatorname{rect}\left(\frac{t-n T_{s} / N-T_{s} / 2 N}{T_{s} / N}\right),
$$

where $T_{s}$ is the sub-pulse duration period. The baseband echoes of $c(t)$ from the target within the interested rangeazimuth bin can be written as

$$
v(t)=\alpha c(t-\tau)+e(t),
$$

where $\alpha$ is a complex number accounting for propagation factor and backscattering factor from the target and $e(t)$ is the sum of the thermal noise and interference. After subpulse matched filtering with the filtering gain $G$, the $N$ dimensional sampling vector $\boldsymbol{v}^{\prime}=\left[v^{\prime}(0), \ldots, v^{\prime}(N-1)\right]^{T} \in$ $\mathbb{C}^{N}$ of the fast-time observations can be expressed as

$$
\boldsymbol{v}^{\prime}=\alpha^{\prime} \boldsymbol{c}+\boldsymbol{e}^{\prime}
$$

with $\alpha^{\prime}=G \alpha$. The vector $c=[c(0), \ldots, c(N-1)]^{T} \in$ $\mathbb{C}^{N}$ represents the fast-time radar sequence and $\boldsymbol{e}^{\prime}=$ $\left[e^{\prime}(0), \ldots, e^{\prime}(N-1)\right]^{T}$ denotes the filtered interference samples. Specifically, in this context, $\boldsymbol{e}^{\prime}$ accounts for white internal thermal noise and the disturbance from the overlaid $\mathrm{FH}$ telecommunication radiation. Without loss of generality, the SINR can be recast by ignoring the constant $\alpha_{t}^{\prime}$ as SINR $=\boldsymbol{c}^{\dagger} \boldsymbol{M}^{-1} \boldsymbol{c}$, where $\boldsymbol{M}=\mathbb{E}\left[\boldsymbol{e} \boldsymbol{e}^{\dagger}\right]$. From the radar detection performance point of view, $c$ is expected to be designed for enhanced SINR according to $\boldsymbol{M}$. In other words, the generated sequence in the algorithm are expected to match the perceived radiation environment.

For the FH communication system, as is shown in Fig. 1, the time-frequency model of the $\mathrm{FH}$ signal is as follows:

$$
f(t)=f_{c}+l\left(n^{\prime}\right) \Delta f \quad\left(n^{\prime} T_{p}<t<\left(n^{\prime}+1\right) T_{p}, n^{\prime} \geq 0\right),
$$

where $f(t)$ is the instantaneous frequency at time $t, f_{c}$ is the carrier frequency, $n^{\prime}$ is the hopping frequency index, $l\left(n^{\prime}\right)$ is a pseudo-random integer sequence, $\Delta f$ is the hopping frequency step length corresponding to the channel interval, and $T_{p}$ is the duration of each frequency hopping period. Actually, $l\left(n^{\prime}\right)$ determines the frequency hopping mode, and without loss of generality, we assume that each channel has the same opportunity to be on line. We consider that there exist multiple bits in the bit stream during one $T_{p}$, i.e., slow frequency hopping (SFH) system.

We assume that there exist $I$ channels in the FH system with a set of $I$ disjoint frequency bands defined by $\Omega=$ I $\bigcup_{i=1}^{I}\left\{f_{i 1}, f_{i 2}\right\}$, where $f_{i 1}$ and $f_{i 2}$ denote the lower and upper normalized frequencies of the $i$ th coexisting frequency

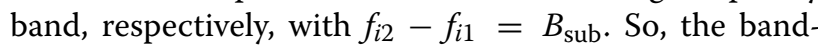

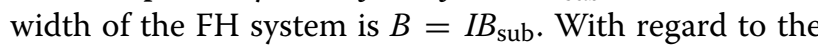
$\mathrm{FH}$ communication system, the bit error ratio (BER) is an important parameter index of data transmission accuracy within the given time.

Multiple frequency-shift keying (MFSK), as one kind of $\mathrm{FH}$ communication modulation form, is a form of $\mathrm{M}$ ary orthogonal modulation, where each symbol consists of one element from an alphabet of orthogonal waveforms. $M$, the size of the alphabet, is usually a power of two so that each symbol represents $\log _{2} M$ bits. Specifically, when $M=2$, BFSK modulation (incoherent case) is employed, and the BER of the $i$ th channel can be given as $[28,29]$ :

$$
\begin{aligned}
\zeta_{i} & =\frac{1}{2} e^{-\rho_{b i} / 2}(i \in[1, \ldots, I]) \\
& =\frac{1}{2} e^{-E_{b} /\left(2 I_{i}\right)}
\end{aligned}
$$




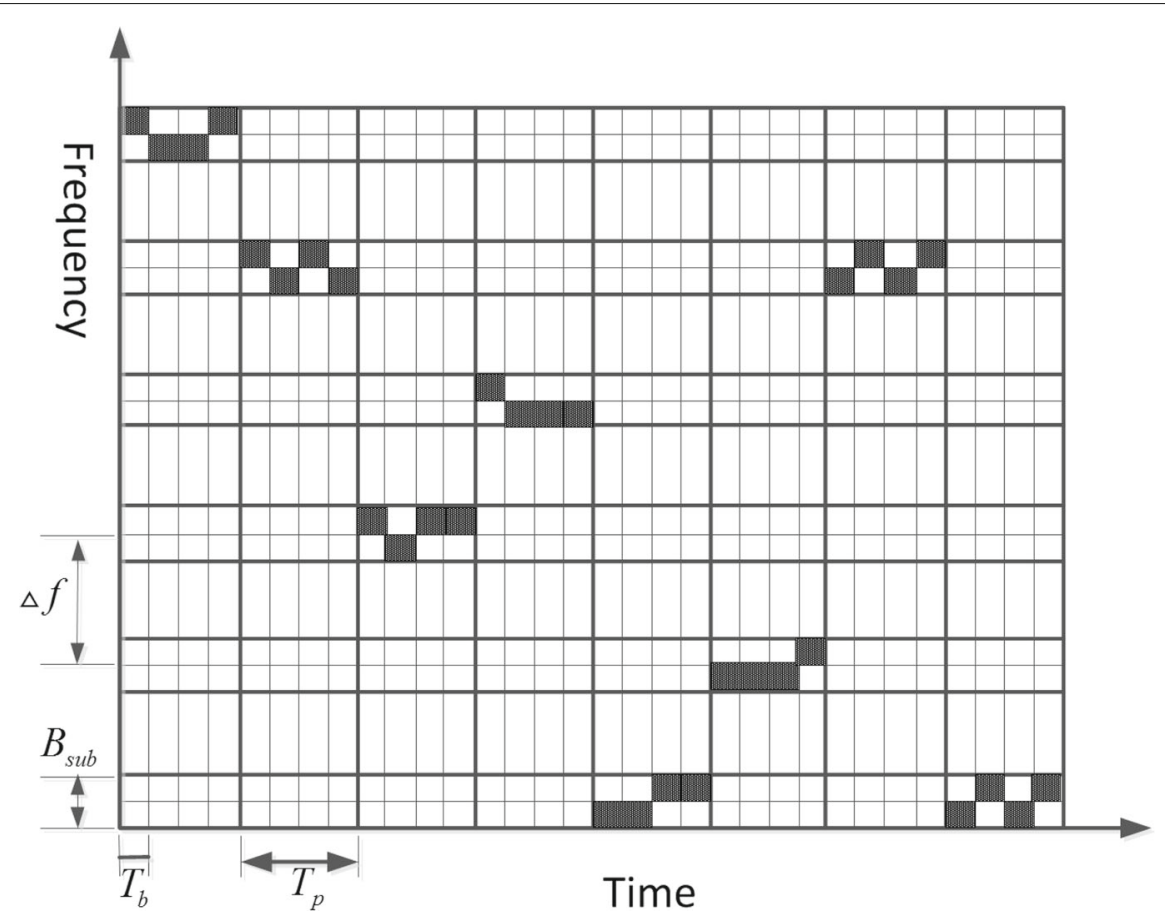

Fig. 1 Slow frequency hopping model

where $E_{b}$ is 1-b energy and $I_{i}$ is the power spectral density (PSD) level of interference in the $i$ th channel. Furthermore,

$$
E_{b}=P_{s} T_{b}=P_{s} / R
$$

where $P_{s}$ is the signal power, $T_{b}$ is the duration of one code, and $R$ is the bit rate and also the symbol rate for BFSK. The PSD of interference is $I_{i}=P_{I} / B_{\text {sub }}$, where $P_{I}$ is the interference power and $B_{\text {sub }}$ is the channel bandwidth. Therefore,

$$
\rho_{b i}=\frac{E_{b}}{I_{i}}=\frac{B_{\mathrm{sub}} / R}{P_{I} / P_{s}}=\frac{B_{\mathrm{sub}} T_{b}}{P_{I} / P_{s}}
$$

For BFSK, $B_{\text {sub }}=2 / T_{b}$, then $B_{\text {sub }} T_{b}=2$, and $\eta_{i}=$ $P_{s} / P_{I}$ is defined as the signal-to-interference ratio (SIR) in the following context, and then we get

$$
\zeta_{i}=\frac{1}{2} e^{-\eta_{i}}(i \in[1, \ldots, I]) \text {. }
$$

For the above Eq. (7), the PSD of interference is assumed to be uniformly distributed over the channel band, whereas it is not the case in practice; therefore, under more general cases, it is given as:

$$
\eta_{i}=\frac{P_{s}}{\int_{f_{i 1}}^{f_{i 2}} p(f) d f}
$$

where $p(f)$ is the power spectrum density (PSD) of the interference.

As the FH system hops on varying channels at each frequency hopping cycle, the use ratio of each channel is the same; hence, the probability of the $i$ th channel in use $p(i)$ is $1 / I$.

The average BER of the FH communication system can be given as:

$$
\begin{aligned}
\zeta & =\sum_{i=1}^{I} p(i) \eta_{i} \\
& =\frac{1}{2 I} \sum_{i=1}^{I} \exp \left(-\eta_{i}\right) \\
& =\frac{1}{2 I} \sum_{i=1}^{I} \exp \left(-\frac{P_{s}}{\int_{f_{i 1}}^{f_{i 2}} p(f) d f}\right) \\
p(f) & =\epsilon \text { for } f \in\left[f_{i 1}, f_{i 2}\right], i \in[1, \\
\zeta & =\frac{1}{2 I} \sum_{i=1}^{I} \exp \left(-\frac{P_{s}}{\epsilon B_{\text {sub }}}\right) \\
& =\frac{1}{2} \exp \left(-\frac{P_{s}}{\epsilon B_{\text {sub }}}\right)
\end{aligned}
$$

If $p(f)=\epsilon$ for $f \in\left[f_{i 1}, f_{i 2}\right], i \in[1, \ldots, I]$, Eq. (10) can be recast into:

In order to facilitate the comparison, the overall SIR is expressed as:

$$
\eta=\frac{I P_{s}}{\sum_{i=1}^{I} \int_{f_{i 1}}^{f_{i 2}} p(f) d f}
$$

From the formula above, $\eta$ is a monotony decrease function of $\zeta$ which shall directly affect the FH communication performance. With all the channels given the same 
weights, the PSD $p(f)$ of each channel has to be under an acceptable level. In other words, the integrated energy on each channel has to be under control.

In order to measure the integrated energy accurately, we define $\Omega$ as the normalized frequency band. Corresponding to $\Omega$, the points of the DFT frequency grid $\bar{N}$ is fixed large enough to cover $\Omega$ densely. $\mathbf{F}_{\bar{N}}$ denotes the $\bar{N} \times \bar{N}$ DFT matrix whose $(m, l)$ th element is given by

$$
\left[\mathbf{F}_{\bar{N}}\right](m, l)=\frac{1}{\sqrt{\bar{N}}} \exp \left\{j 2 \pi \frac{(m-1)(l-1)}{\bar{N}}\right\}
$$

where $m, l \in(1, \ldots, \bar{N})$ and the coefficient $1 / \sqrt{\bar{N}}$ makes sure $\mathbf{F}_{\bar{N}}$ to be unitary. Accordingly, $\overline{\boldsymbol{c}}$, the extended sequence of $\boldsymbol{c}$, is defnied as

$$
\overline{\boldsymbol{c}}=[c(0) \cdots c(N-1) \underbrace{0 \cdots 0}_{\bar{N}-N}]^{T} .
$$

For calculating the energy integrated on each stopband (or so-called channel), we define the matrix $S_{i}$, $(i=$ $1, \ldots, I)$, which shall be obtained from the combined columns of $\mathbf{F}_{\bar{N}}$ corresponding to the $i$ th band $\Omega_{i}$ in $\Omega$. For instance, with the whole normalized spectrum $\Omega=$ $[-0.5,0.5], \bar{N}=1000$, if $\Omega_{1}=[0.1,0.11]$, then $S_{1}$ is combined by the columns from 100th to 200th in $\mathbf{F}_{\bar{N}}$ (indexed from 0). In another way, $\boldsymbol{S}_{1}$ is a sub-matrix of $\mathbf{F}_{\bar{N}}$ with the size $1000 \times 101$. Besides, the column index vector ([600, 601, .., 610]) can be noted as $\Omega_{d, i}$. In this way, the problem of suppressing the spectral power of the waveform over the $i$ th band can be converted to minimizing the following criterion

$$
\left\|\boldsymbol{S}_{i}^{\dagger} \bar{c}\right\|^{2} \text {. }
$$

The formula in (15) can be extended as

$$
\begin{aligned}
\left\|S_{i}^{\dagger} \bar{c}\right\|^{2} & =\overline{\boldsymbol{c}}^{\dagger} \boldsymbol{S}_{i} \boldsymbol{S}_{i}^{\dagger} \overline{\boldsymbol{c}} \\
& =[\boldsymbol{c} ; \mathbf{0}]^{\dagger} \boldsymbol{S}_{i} \boldsymbol{S}_{i}^{\dagger}\left[\begin{array}{c}
\boldsymbol{c} \\
\mathbf{0}
\end{array}\right]=[\boldsymbol{c} ; \mathbf{0}]^{\dagger} \overline{S_{i}}\left[\begin{array}{l}
\boldsymbol{c} \\
\mathbf{0}
\end{array}\right] \\
& =\boldsymbol{c}^{\dagger} \tilde{\boldsymbol{S}}_{i} \boldsymbol{c},
\end{aligned}
$$

where $\overline{S_{i}}=S_{i} S_{i}^{\dagger}$ and $\tilde{S}_{i}=\overline{S_{i}}(1: N, 1: N)$. The total integrated interference energy over the FH channels can be given as:

$$
E=c^{\dagger} \bar{S} \boldsymbol{c}
$$

with $\bar{S}=\sum_{i=1}^{I} \overline{S_{i}}$.

In order to improve the SINR of the radar system and an acceptable BER of HP communication system, it is necessary to modulate the spectrum of radar sequences to make sure that the integrated energy over the frequency hopping channels is under acceptable level. In this way, the desired discrete spectrum is given as:

$$
P_{T}(k)=\left\{\begin{array}{c}
\epsilon_{i} k \in \Omega_{d, i}, k \in[1, \ldots, \bar{N}], i \in[1, \ldots, I] \\
1 k \notin \Omega_{d, i}, k \in[1, \ldots, \bar{N}], i \in[1, \ldots, I]
\end{array},\right.
$$

where

$$
\epsilon_{i}=\epsilon=\frac{P_{s}}{-B_{\text {sub }} \ln (2 \zeta)}
$$

according to Eq. (11).

When the PSD of the generated sequence approaches $P_{T}$ in the iteration process,

$$
\sum_{k=1}^{\bar{N}}\left|P(k)-P_{T}(k)\right|^{2}
$$

will be small enough.

The ACF sidelobes of the generated sequence are expected to be under required levels, in order to avoid the weak targets being masked by the sidelobes of strong targets. The desired waveform should own low PSL of ACF to avoid interference of echoes among different range bins.

The most concerned sidelobes are $r(n), n \in \Psi \triangleq$ $\{ \pm 1, \pm 2, \cdots, \pm N\}$, and the template for the desired sidelobe level is

$$
r_{T}(n)=\left\{\begin{array}{cc}
p_{n} \mathrm{~dB} & n \in \Psi \\
0 \mathrm{~dB} & n \notin\{0\} \cup \Psi
\end{array},\right.
$$

where $p_{n}$ is the desired sidelobe level for the sidelobes in $\Psi$.

The figure of merit accounting for the similarity between the actual ACF and the desired one is given by:

$$
\sum_{n=1}^{N}\left|r(n)-r_{T}(n)\right|^{2}
$$

This optimum problem requires minimizing the sidelobe levels on the premise that the PSD over the HP channels are below the given level. As a whole, the waveform design problem can be expressed as:

$$
\mathrm{P}_{1}\left\{\begin{array}{ll}
\min _{c} & \sum_{n=1}^{N}\left|r(n)-r_{T}(n)\right|^{2} \\
\text { s.t. } & |c(n)|=1, n=0, \ldots, N-1, \\
& \sum_{k=1}^{N}\left|P(k)-P_{T}(k)\right|^{2}<\xi
\end{array},\right.
$$

As the optimum problem considers both the PSD and ACF terms with PSD requirements as the necessary one and $\mathrm{ACF}$ requirements as the optimal one, it can not be treated by Lagrange multiplier method because the problem is difficult to take the derivative. We use another method to resolve this problem. Defining $f_{p}=$ $\sum_{k=1}^{\bar{N}}\left|P(k)-P_{T}(k)\right|^{2}$, in this way, the original problem can be converted as:

$$
\mathrm{P}_{2}\left\{\begin{array}{l}
\min _{c} \lambda\left(f_{p}\right) f_{p}+\left(1-\lambda\left(f_{p}\right)\right) \sum_{n=1}^{N}\left|r(n)-r_{T}(n)\right|^{2} \\
\text { s.t. } \quad|c(n)|=1, n=0, \ldots, N-1
\end{array},\right.
$$


where $\lambda$, adjusted according to $f_{p}$, is the weight between the spectrum compatibility and sidelobe suppression. $\lambda$ is adjusted to meet the PSD requirements with higher priority than ACF requirements. When the PSD requirements are not satisfied in the iteration procedure, $\lambda$ will increase by tiny step length in each iteration until they are satisfied or $\lambda$ comes to its maximum value allowed.

The optimum problem can be expressed more simplified as:

$$
\mathrm{P}_{3}\left\{\begin{array}{l}
\min _{c} \lambda\left(f_{p}\right)\left\|\boldsymbol{P}-\boldsymbol{P}_{T}\right\|^{2}+\left(1-\lambda\left(f_{p}\right)\right)\left\|\boldsymbol{r}-\boldsymbol{r}_{T}\right\|^{2}, \\
\text { s.t. }|c(n)|=1, n=0, \ldots, N-1
\end{array},\right.
$$

with

$$
\begin{aligned}
& \boldsymbol{P}=[P(1), P(2), \ldots, P(\bar{N})], \\
& \boldsymbol{P}_{T}=\left[P_{T}(1), P_{T}(2), \ldots, P_{T}(\bar{N})\right], \\
& \boldsymbol{r}=[r(1), r(2), \ldots, r(N)], \\
& \boldsymbol{r}_{T}=\left[r_{T}(1), r_{T}(2), \ldots, r_{T}(N)\right] .
\end{aligned}
$$

\section{Generalized fitting template algorithm}

Before the algorithm statement, one reference sequence should be given as the original sequence. As the linear frequency modulated (LFM) waveforms own good Doppler tolerance character and low sidelobes, an instance is employed for reference as follows:

$$
c_{0}(n)=e^{j \pi K_{s}\left(n / f_{s}\right)^{2}} / \sqrt{N} \quad n=0, \ldots, N-1,
$$

where $K_{s}$ is the slope. The extended reference sequence is

$$
\overline{\boldsymbol{c}}_{0}=[c_{0}(0) \cdots c_{0}(N-1) \underbrace{0 \cdots 0}_{\bar{N}-N}]^{T} \text {. }
$$

In the iteration process, $\overline{\boldsymbol{c}}_{l}$ denotes the generated sequence after the $l$ th iteration.

Its frequency spectrum is calcualted as:

$$
\begin{aligned}
S_{l}[k] & =\sum_{n=0}^{\bar{N}-1} \bar{c}_{l}[n] e^{-j 2 \pi n k / \bar{N}} \\
& =\left|S_{l}[k]\right| e^{j \angle\left(S_{l}[k]\right)}, k \in[0, \ldots, \bar{N}-1] .
\end{aligned}
$$

Accordingly, $\boldsymbol{r}_{l}$ is the ACF vector of $\overline{\boldsymbol{c}}_{l}$ after the $l$ th iteration, and $r_{l}[n]$ denotes the $n$th element of $\boldsymbol{r}_{l}$ :

$$
r_{l}(n)=\left|r_{l}(n)\right| e^{j \angle\left(r_{l}(n)\right)}, n \in[0, \ldots, \bar{N}-1] .
$$

The amplitude spectrum of $\boldsymbol{r}_{l}$, namely $P_{l}(k)$, is given as:

$$
P_{l}(k)=\left|\sum_{n=0}^{\bar{N}-1} r_{l}[n] e^{-j 2 \pi n k / \bar{N}}\right|, k \in[0, \ldots, \bar{N}-1]
$$

Furthermore, for the input parameters, $\lambda_{0}, \lambda_{T_{u}}, \lambda_{T_{d}}$, and $\Delta$ denote the initial value, the upper bound, the lower bound, and the step length of $\lambda$, respectively.

$\xi$ is defined as the similarity threshold for the PSD of the generated sequence. Based on $\xi$, the algorithm adjusts the weight coefficient $\lambda$ adaptively in the iteration process to meet the algorithm goals. $\rho \in(0,1)$ is defined as the update coefficient to fix the updating step length of the ACF features of the generated sequences. Moreover, we define the two-norm tolerance $\delta$ as the threshold for ending the iteration.

Description of the generalized fitting template (GFT) algorithm:

Input: $\overline{\boldsymbol{c}}_{0}, \boldsymbol{P}_{T}, \boldsymbol{r}_{T}, \lambda_{0}, \lambda_{T_{u}}, \lambda_{T_{d}}, \Delta, \xi, \rho, \delta$.

Output: A convergence solution $\boldsymbol{c}_{\text {out }}$.

1. For the iteration index $l=0$, prepare $\boldsymbol{S}_{0}, \boldsymbol{P}_{0}$, and $\boldsymbol{r}_{0}$ by (29), (30), and (31).

2. For the iteration index $l \geq 1$, get the new power spectrum $\boldsymbol{P}_{s}$ by:

$P_{s}(k)=\left\{\begin{array}{l}\left|P_{T}(k)\right| \quad k \in \Omega_{d, i}, k \in[1, \ldots, \bar{N}], i \in[1, \ldots, I] \\ \left|P_{l-1}(k)\right| k \notin \Omega_{d, i}, k \in[1, \ldots, \bar{N}], i \in[1, \ldots, I]\end{array}\right.$,

$$
\boldsymbol{P}_{s}=\left[P_{s}(0), \ldots, P_{s}(\bar{N}-1)\right] .
$$

3. IF $l \geq 100$

$$
\begin{aligned}
& \text { Calculate } f_{p}(l)=\sum_{k=1}^{\bar{N}}\left|P_{l}(k)-P_{T}(k)\right|^{2} ; \\
& \text { IF } f_{p}(l)>\xi \\
& \text { IF } \lambda(l)<\lambda T_{u} \\
& \lambda(l)=\lambda(l)+\Delta \\
& \operatorname{END} \\
& \operatorname{ELSEIF~} f_{p}(l)<0.9 \times \xi \\
& \operatorname{IF} \lambda(l)>\lambda_{T_{d}} \\
& \lambda(l)=\lambda(l)-\Delta \\
& \operatorname{END} \\
& \text { END }
\end{aligned}
$$

END

4. Calculate the ACF of $\overline{\boldsymbol{c}}_{l-1}$ by inverse fast Fourier transform (IFFT) as $\boldsymbol{r}_{l-1} \overleftarrow{\mathrm{IFFT}} \boldsymbol{P}_{l-1}$, obtain the phase by $e^{j \angle \boldsymbol{r}_{l-1}}$ and then figure out the new ACF by:

$$
\begin{aligned}
r_{a}(n)= & \left(\rho \times\left|r_{l-1}(n)\right|+(1-\rho) \times \min \left(\left|r_{l-1}(n)\right|,\left|r_{T}(n)\right|\right)\right) \\
& \times e^{j<\left(r_{l-1}(n)\right)}, n \in[0, \bar{N}-1] .
\end{aligned}
$$

5. Generate the power spectrum corresponding to $\boldsymbol{r}_{a}$ by fast Fourier transform (FFT) as $\boldsymbol{P}_{a} \overleftarrow{\text { FFT }} \boldsymbol{r}_{a}$ with $\boldsymbol{r}_{a}=$ $\left[r_{a}(0), \ldots, r_{a}(\bar{N}-1)\right]$ and $\boldsymbol{P}_{a}=\left[P_{a}(0), \ldots, P_{a}\right.$ $(\bar{N}-1)]$.

6. Calculate the adjusted amplitude spectrum by:

$$
\begin{gathered}
F_{\text {sum }}(k)=\lambda(l) \sqrt{\mid P_{s}(k)}+(1-\lambda(l)) \sqrt{P_{a^{\prime}}(k)}, \\
k \in[0, \ldots, \bar{N}-1]
\end{gathered}
$$


with

$P_{a^{\prime}}(k)=\left\{\begin{array}{c}\left|P_{T}(k)\right| k \in \Omega_{d, i}, k \in[1, \ldots, \bar{N}], i \in[1, \ldots, I] \\ \left|P_{a}(k)\right| k \notin \Omega_{d, i}, k \in[1, \ldots, \bar{N}], i \in[1, \ldots, I]\end{array}\right.$,

7. Get the new frequency spectrum $F_{t}(k)$ by:

$$
\begin{aligned}
F_{t}(k) & =\left|F_{\text {sum }}(k)\right| e^{j \angle\left(\bar{c}_{l-1}(k)\right)}, k \in[0, \ldots, \bar{N}-1] \\
\boldsymbol{F}_{t} & =\left[F_{t}(0), \ldots, F_{t}(\bar{N}-1)\right]
\end{aligned}
$$

8. By taking IFFT operation to $\boldsymbol{F}_{t}$ and keeping only the phase information, the new waveform $\overline{\boldsymbol{c}}_{t}$ can be generated as:

$$
\overline{\boldsymbol{c}}_{l}=e^{j \measuredangle\left(\operatorname{IFFT}\left(\boldsymbol{F}_{t}\right)\right)} ;
$$

9. Calculate $\nabla=|f(l)-f(l-1)|$ with $f(l)=\lambda(l)\left\|\boldsymbol{P}_{l}-\boldsymbol{P}_{T}\right\|_{2}+(1-\lambda(l))\left\|\boldsymbol{r}_{l}-\boldsymbol{r}_{T}\right\|_{2}$. If $\nabla>\delta$, then repeat the iteration from 2-nd step to 7-th step, otherwise, output $\boldsymbol{c}_{\text {out }}=\overline{\boldsymbol{c}}_{l}(1: N)$.

For the iteration process, the motivation for each step is given as follows:

\section{Initialization:}

For the input parameters, the typical value of $\lambda_{0}, \lambda_{T_{u}}$, $\lambda_{T_{d}}, \Delta, \rho, \delta$ are $0.6,0.9,0.2,0.001,0.7,0.001$, respectively. $\xi$ is decided by the required BER of $F H$ system. Step (1) prepares the initial values of intermediate variables.

2. Spectrum Modulation by PSD Template: Step (2) modulates the power spectrum of generated sequence of last iteration to approach the PSD template.

3. Weight Adjustment ( $\lambda$ ):

Step (3) adjusts the weight $\lambda$ adaptively according to the relationship between the function value $f_{p}(l)$ and the similarity threshold $\xi$.

4. Spectrum Modulation by ACF Template: Steps (4)-(5) modulate the power spectrum of generated sequence of last iteration as $\boldsymbol{P}_{\boldsymbol{a}}$ according to ACF template. $\rho(0<\rho<1)$ is the update coefficient. Smaller $\rho$ indicates larger update speed, but it must be chosen suitably and cannot be small arbitrarily to affect the convergence.

5. Amplitude Spectrum Synthesis:

Step(6) combines the amplitude spectrums that take along both PSD and PSL template requirements. to approach the PSD template.
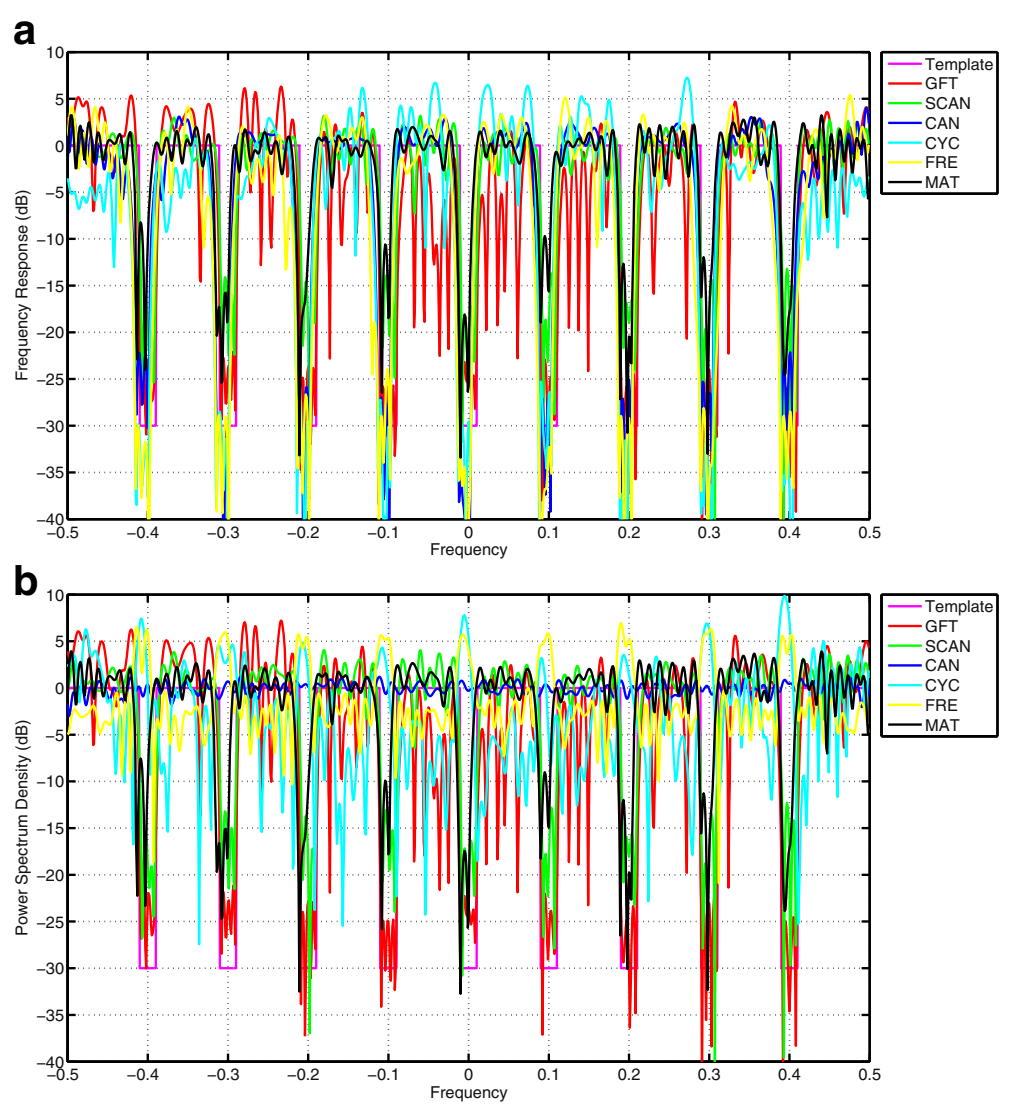

Fig. 2 a Frequency response. b PSD performance 


\section{Frequency Spectrum Synthesis:}

Step (7) obtains the new frequency spectrum by combining the amplitude spectrum in Step (6) and the phase information of $\overline{\boldsymbol{c}}_{l-1}$.

7. New Sequence Generation:

Step (8) generates the new unimodular sequence code.

8. Iteration Termination:

Step (9) gives judgment for finishing the iteration process.

\section{Simulation results and analysis}

For the simulation settings, the sequence length is $N=$ 100 , the extended sequence length is $\bar{N}=1000$, and the operation bandwidth is $6 \mathrm{MHz}$. The reference sequence is defined as in (27), with $K_{s}=3.75 \times 10^{9} \mathrm{~Hz} / \mathrm{s}$ and $f_{s}=800$ $\mathrm{KHz}$. It is noteworthy that, in order to compare the compatibility performance more clearly among the following six algorithms, the SINRs for both radar and FH system are replaced by SIRs in the following simulations.

In the following, we give some simulation results from different aspects and compare the performance of GFT with that of SCAN, CAN, CYC, FRE, and MAT algorithms [30].

\subsection{Frequency response and PSD performance}

Figure 2a shows the frequency responses of the six algorithms' receiver filters, respectively. The channel number is 9, and channel bandwidth is $B_{\text {sub }}=0.02$. The filters of GFT, SCAN, and MAT are matched filters, whereas that of CAN, CY, and FRE are mismatched cases. As the communication signals from the HF system affects the SINR at the radar receiver, a deeper suppression depth of frequency response on each channel indicates a higher SIR for radar system.

Figure 2a shows that the interference suppression level of filter generated by MAT is not stable for different channels. That of SCAN is during $[-15,-20] \mathrm{dB}$. GFT achieves about $-25 \mathrm{~dB}$ in average. CYC and FRE behave slightly better than CAN and GFT, achieving about $-30 \mathrm{~dB}$ in average, whereas the former three algorithms show narrower suppression bandwidth than that of GFT by about

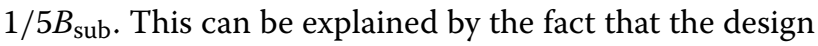
of filters for CYC and FRE which have no unimodular restrains, therefore, are given higher degrees of freedom than that of GFT. Whereas, the extended length $\bar{N}$, involoved in the DFT operation by GFT algorithm, covers the spectrum space much more densely than the other three algorithms.

Figure $2 \mathrm{~b}$ shows the PSD of the sequences generated by the considered six algorithms. Based on the required BER level, the required average PSD level of the generated sequence for each channel (corresponding to each shared frequency stop-bands) is fixed at $-30 \mathrm{~dB}$. The
PSD of MAT within the shared frequency stop-bands behaves unsteadily and irregularly; besides, the suppression bandwidth is much more narrow than the required bandwidth. SCAN can achieve at least $-15 \mathrm{~dB}$, but at most $-20 \mathrm{~dB}$, whereas the spectrums of the generated sequence by CAN, CYC, and FRE show no suppression abilities on the stop-bands, because these three algorithms optimize the SIR of the radar system by designing the receive filters adaptively. For CYC and FRE, the PSD levels within the stop-bands are even higher than those out of the stopbands. It is fair to say that GFT behaves better than the other five algorithms, and it almost achieves the required level: the PSD comes to at least $-24 \mathrm{~dB}$, at most $-27 \mathrm{~dB}$ for each channel in average.

\subsection{PSD and PSL convergence performance}

Figure 3 shows the iteration process of PSD and PSL with fixed BER requirements from the FH system. The PSD levels of all $\mathrm{FH}$ channels are required below $-25 \mathrm{~dB}$ to ensure an acceptable BER. In Fig. 3a, the PSD index shows rapid convergence to about $-26 \mathrm{~dB}$ after about 1000 iterations,

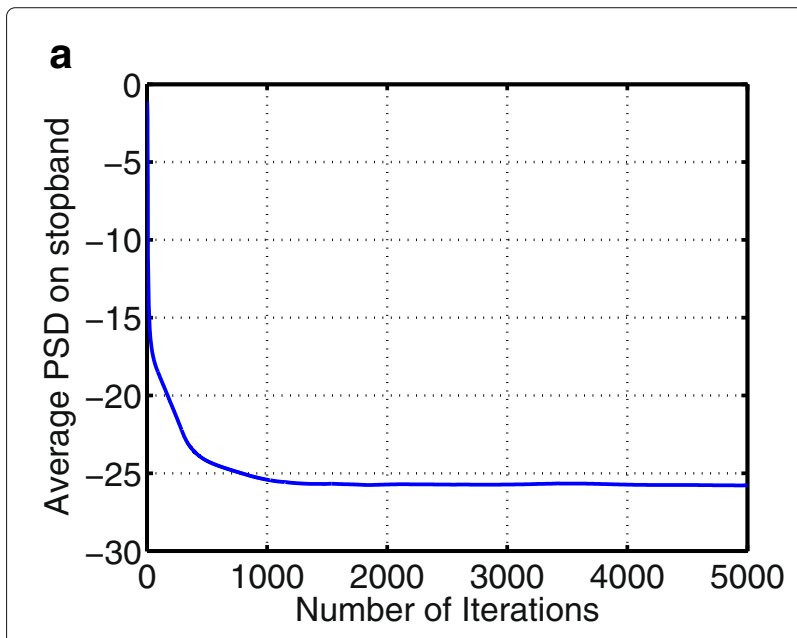

b

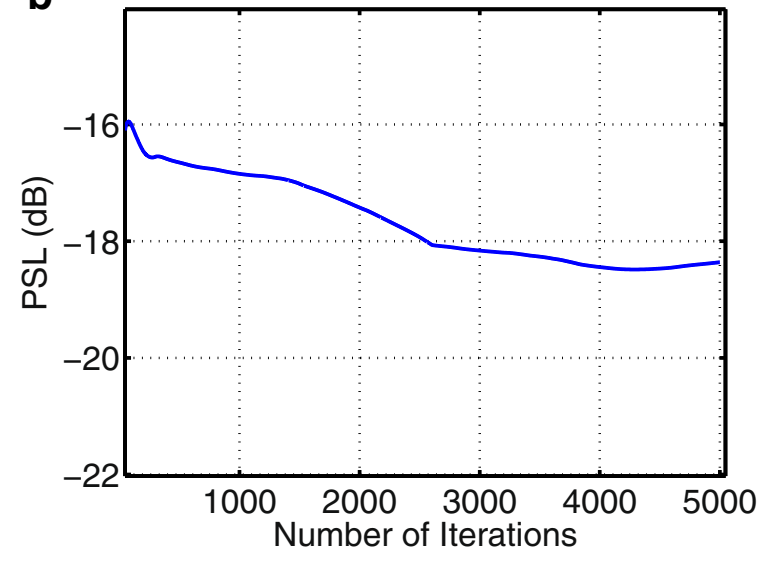

Fig. 3 a, b PSD and PSL iteration performance 


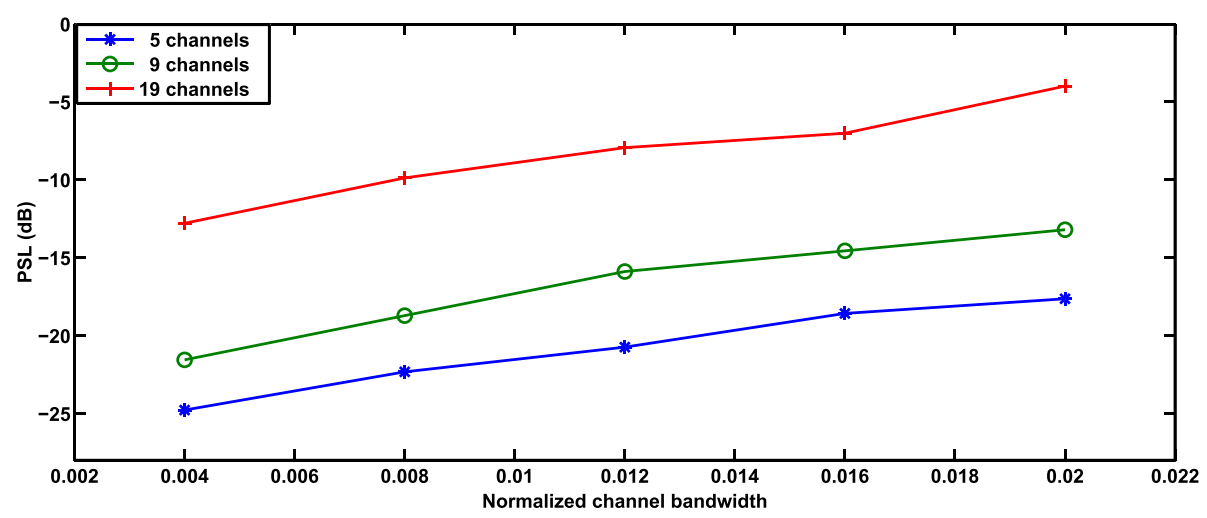

Fig. 4 PSL perfromace versus channel bandwidth $B_{\text {sub }}$

whereas in Fig. 3b, the PSL level decreases slowly because the priority of the weight of PSL in the algorithm is lower than that of the PSD to ensure the compatibility requirements at first in the iteration process. After the compatibility requirements are met, the algorithm shall give the PSL term greater weight to improve PSL.

\subsection{PSL performance versus channel bandwidth and BER}

Figure 4 shows the PSL behavior versus channel number and channel bandwidth under fixed BER requirement. The figure indicates that the PSL increases monotonically with $B_{\text {sub }}$. Besides, with fixed $B_{\text {sub }}$, more channels, although provide more degrees of freedom for the $\mathrm{FH}$ communication system, however, indicate higher PSLs of the generated sequences.

Figure 5 shows the PSL versus required BER and the channel number. With higher required BER level, the PSLs can be restrained with lower levels, whereas for fixed BER requirements, the PSL levels increase monotonically with the FH channel number. The phenomenon shows the competitive relationship clearly between spectrum modulation and sidelobe levels suppression. Ideal sidelobe levels require whiten spectrum, which indicates mutual independence among all codes in the sequence.

\subsection{SIR of radar and FH system}

Figure 6 shows the SIR for the radar system of the six algorithms versus channel number and $B_{\text {sub. }} B_{\text {sub }}$ is set at 0.02 and 0.04, respectively. The SIR of GFT achieves at least $33.5 \mathrm{~dB}$, at most $39.2 \mathrm{~dB}$ when $B_{\mathrm{sub}}=0.02$; it comes to at least $33 \mathrm{~dB}$, at most $37 \mathrm{~dB}$ when $B_{\text {sub }}=0.04$. Whereas for all of the other 5 algorithms, the SIR achieves at least $16.5 \mathrm{~dB}$, at most $27 \mathrm{~dB}$ when $B_{\text {sub }}=0.02$, it reaches at least $13 \mathrm{~dB}$, at most $26 \mathrm{~dB}$ when $B_{\mathrm{sub}}=0.04$. The SIR of GFT behaves at least $6 \mathrm{~dB}$ and at most $22 \mathrm{~dB}$ better than that of the other five algorithms. The phenomenon can be explained by the fact that GFT algorithm gives higher priority to spectrum compatibility requirement to suppress the interference energy. Besides, the filters of CAN, CYC, and FRE are mismatched filters, which cause mismatch loss at the receiver ends and therefore decrease the SIR.

Figure 7 shows the SIR for the FH system with the same parameter settings as in Fig. 6. All six algorithms show relative stable performance no matter what the channel number and bandwidth are. As CAN, CYC, and FRE algorithms consider the joint design for waveform and receiver filter to restrain the interference from the $\mathrm{FH}$ communication system, the spectrums of generated sequences of these algorithms are not compatible with the spectrum

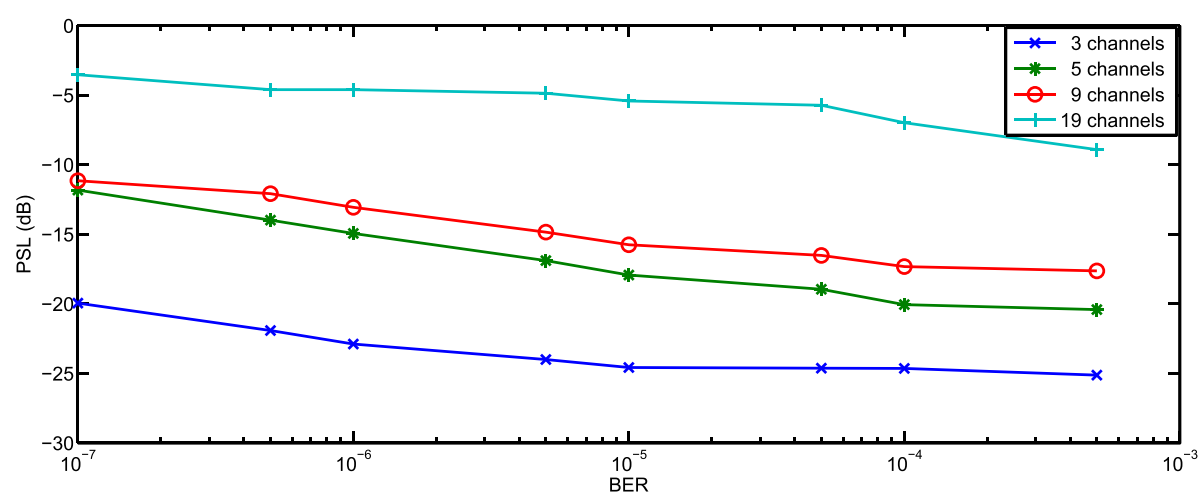

Fig. 5 PSL perfromace versus BER 


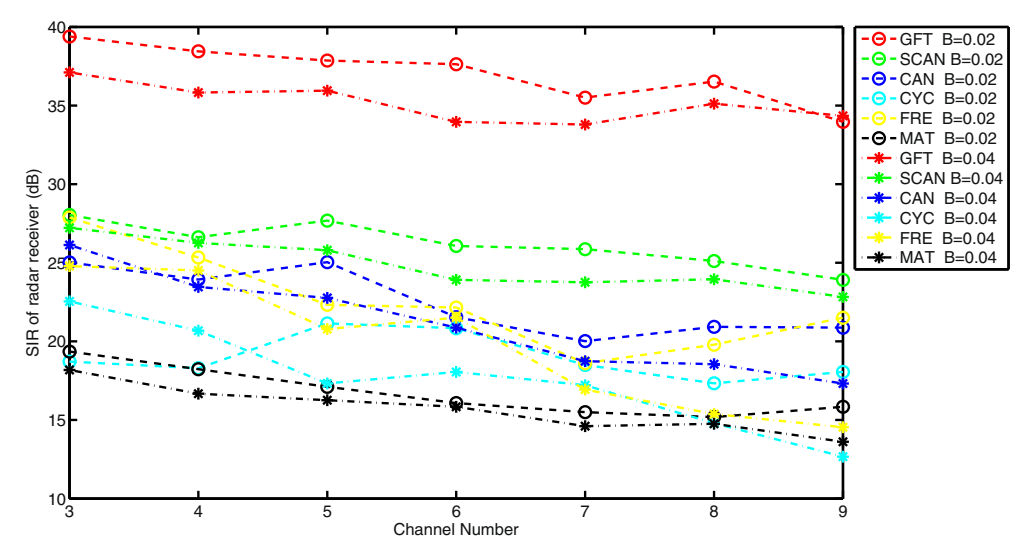

Fig. 6 SIR of radar versus channel number and bandwidth

requirements of the $\mathrm{FH}$ communication. In another way, these three algorithms do not care about the interference from the radar to the FH communication system. The SIRs for the FH system of them are all below the acceptable level, namely $1 \mathrm{~dB}$. The SIR of the SCAN achieves about at least $15 \mathrm{~dB}$, at most $17 \mathrm{~dB}$ when $B_{\text {sub }}=0.02$; it comes to at least $16.5 \mathrm{~dB}$, at most $17.5 \mathrm{~dB}$ when $B_{\text {sub }}=0.04$. The SIR of MAT achieves about $6.5 \mathrm{~dB}$ when $B_{\text {sub }}=0.02$ and $7.8 \mathrm{~dB}$ when $B_{\text {sub }}=0.02$, whereas the SIR of GFT achieves at least $24.5 \mathrm{~dB}$, at most $27 \mathrm{~dB}$ for both bandwidths cases. It is stable and has a high level no matter what the channel number and bandwidth are. The adaptive weight gives strong support to these features of GFT.

\subsection{BER performance comparison}

According to the proceeding discussion, the SIR for $\mathrm{FH}$ system of CAN, CYC, and FRE algorithms are less than $1 \mathrm{~dB}$, which is the lowest acceptable threshold. We mainly compare the performance between GFT, SCAN, and MAT algorithms.
The parameter setting is as follows. The channel number is 9 , the normalized channel bandwidth is 0.02 , and the normalized PSD of FH signal spectrum in each channel is $0 \mathrm{~dB}$, the same as that of the radar signal spectrum out of the stop-bands.

Table 1 shows the BER of GFT, SCAN, and MAT algorithms versus different channel numbers and bandwidths. MAT shows overall the highest BER level compared with SCAN and GFT, no matter what the channel number and channel bandwidth are. The BER of SCAN is lower about two orders than that of MAT as a whole but higher two orders than that of GFT. The BER of GFT is relatively stable regardless of the values of the channel number and bandwidth. This feature benefits from the algorithm characteristic that gives to the mutual compatibility requirement a higher priority.

\subsection{Calculation effectiveness performance}

Our algorithms have been implemented in a MATLAB $2010 \mathrm{~b}$ environment, and the simulations are conducted on

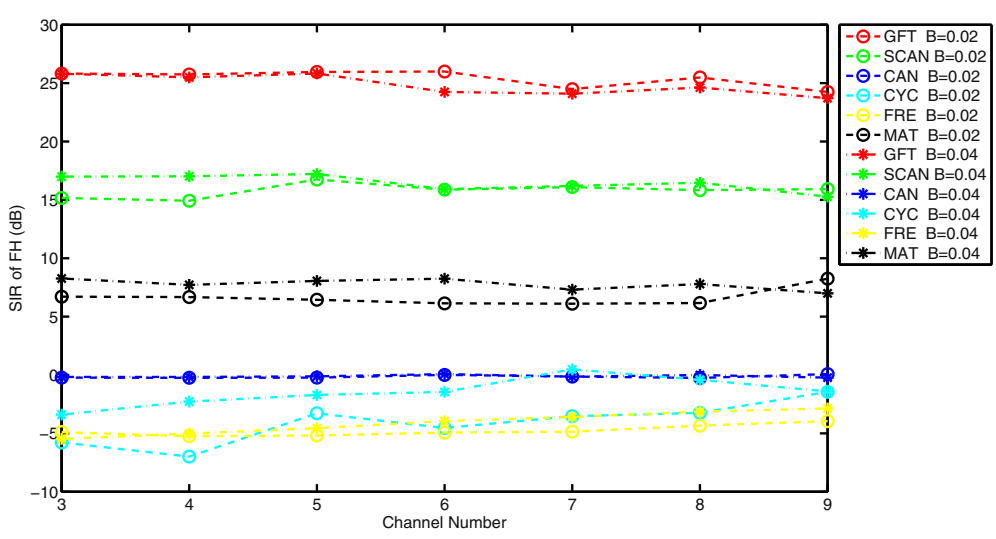

Fig. 7 SIR of FH versus channel number and bandwidth 
Table 1 BER

\begin{tabular}{|c|c|c|c|c|c|c|c|}
\hline Channel number & 3 & 4 & 5 & 6 & 7 & 8 & 9 \\
\hline MAT $B=0.02$ & 0.052 & 0.053 & 0.059 & 0.069 & 0.070 & 0.068 & 0.023 \\
\hline $\mathrm{SCAN} B=0.02$ & $7.64 E-4$ & $8.61 \mathrm{E}-4$ & $3.46 \mathrm{E}-4$ & $5.37 E-4$ & $4.82 E-4$ & $5.45 E-4$ & $5.23 E-4$ \\
\hline $\mathrm{GFT} B=0.02$ & $3.71 E-6$ & $3.86 \mathrm{E}-6$ & $3.46 E-6$ & $3.39 E-6$ & $7.21 E-6$ & $4.39 E-6$ & $1.01 E-5$ \\
\hline MAT $B=0.04$ & 0.024 & 0.031 & 0.027 & 0.024 & 0.038 & 0.030 & 0.045 \\
\hline $\mathrm{SCAN} B=0.04$ & $3.07 E-4$ & $3.02 E-4$ & $2.73 E-4$ & $5.23 E-4$ & $4.57 \mathrm{E}-4$ & $3.99 E-4$ & $7.73 E-4$ \\
\hline $\mathrm{GFT} B=0.04$ & $3.74 \mathrm{E}-6$ & 4.37E-6 & $3.69 E-6$ & $8.14 \mathrm{E}-6$ & $8.80 E-6$ & $6.74 \mathrm{E}-6$ & $1.06 \mathrm{E}-5$ \\
\hline
\end{tabular}

a Window 7 64-b operation system with 2.7-GHz CPU and 6-GB RAM.

Table 2 shows the calculation loads of these six algorithms. Although the time costs of the CAN, CYC, and MAT algorithms are much less than that of the GFT algorithm, the spectrums of generated codes produced by these algorithms are far from satisfactory for spectrum compatibility requirements. Besides, the extended length of sequence is 1000 for GFT and SCAN algorithms, while there exist no extended sequence for the other four algorithms. This is the main reason for the larger computational cost of these two algorithms. Furthermore, the time cost of GFT algorithm is linear with the iteration number. When the design requirements are easy to tackle, the iteration time cost will decrease accordingly.

\section{Conclusions}

This paper proposed a unimodular sequence design technique considering spectrum compatibility and the PSL restrain. Spectrum compatibility is considered between radar system and $\mathrm{FH}$ communication system. It employs generalized template technique to induce the PSD and PSL of designed sequence tending to convergence to that of templates in the iteration process. The PSD template should be designed according to acceptable BER requirement of $\mathrm{FH}$ communication systems.

In order to achieve the BER requirements of FH system with a higher priority, the weight for PSD and PSL in the objective function is adaptively adjusted in the iteration process. The average PSD levels show fast convergence and approach the desired level, whereas the PSLs show slow convergence because of lower priority. The performance of the proposed method is compared with that of SCAN, CAN, CYC, FRE, and MAT algorithms about PSD, frequency response, SIR for both radar and FH communication systems, and BER items, which shows its feature and clear effectiveness. A possible future research track might concern the fusion of wireless communication and

Table 2 Calculation load

\begin{tabular}{llllll}
\hline GFT & SCAN & CAN & CYC & FRE & MAT \\
\hline $9.9 \mathrm{~s}$ & $56.53 \mathrm{~s}$ & $0.53 \mathrm{~s}$ & $2.63 \mathrm{~s}$ & $9.53 \mathrm{~s}$ & $0.16 \mathrm{~s}$ \\
\hline
\end{tabular}

radar systems [31-35], to achieve enhanced LPI performance for communication system with low loss of radar target detection performance.

\section{Acknowledgements}

This work was supported by the National Natural Science Foundation of China under Grants 61201276, 61178068, and 61301266, the Fundamental Research Funds of Central Universities under Grants ZYGX2014J013 and ZYGX2014Z005, the Chinese Postdoctoral Science Foundation under Grant 2014M550465, and the Program for New Century Excellent Talents in University under Grant A1098524023901001063.

\section{Competing interests}

The authors declare that they have no competing interests.

Received: 9 August 2016 Accepted: 25 November 2016

Published online: 28 December 2016

\section{References}

1. M Wicks, in 2010 2nd International Workshop on Cognitive Information Processing (CIP). Spectrum Crowding and Cognitive Radar (IEEE, Elba Island, 2010), pp. 452-457

2. JR Guerci, Cognitive Radar, The Knowledge-Aided Fully Adaptive Approach (Artech House, London, 2010)

3. S Haykin, Cognitive Radar: A Way of The Future. IEEE Trans. Sig. Process. 23(1), 30-40 (2006)

4. S Haykin, Cognitive Dynamic Systems: Radar, Control, and Radio [Point of View]. Proc. IEEE. 100(7), 2095-2103 (2012)

5. Y Zhao, J Gaeddert, KK Bae, JH Reed, in Proceedings of Software Defined Radio (SDR) Technical Conference. Radio Environment Map-enabled Situation-aware Cognitive Radio Learning Algorithms (IEEE, Orlando, 2006)

6. Y Zhao, L Morales, J Gaeddert, in 2007 2nd IEEE International Symposium on New Frontiers in Dynamic Spectrum Access Networks. Applying Radio Environment Maps to Cognitive Wireless Regional Area Networks (IEEE, Dublin, Ireland, 2007), pp. 115-118

7. LS Wang, JP McGeehan, C Williams, A Doufexi, Application of Cooperative Sensing in Radar - Communications Coexistence. IET Radar Sonar Navig. 2(6), 856-868 (2008)

8. A De Maio, F Gini, L Patton, Waveform Design and Diversity for Advanced Radar Systems. (The Institution of Engineering and Technology, London, 2012)

9. JD Taylor, Ultra-Wideband Radar Technology. (CRC Press Inc, Boca Raton, 2001)

10. K Gerlach, Thinned Spectrum Ultrawideband Waveforms Using Stepped-Frequency Polyphase Codes. IEEE Trans. Aerosp. Electron. Syst. 34(4), 1356-1361 (1998)

11. IW Selesnick, SU Pillai, Chirp-Like Transmit Waveforms with Multiple Frequency-Notches. 2011 IEEE Radar Conference, 1106-1110 (2011). Kansas, MO, USA, 23-27 May

12. MJ Lindenfeld, Sparse Frequency Transmit and Receive Waveform Design. IEEE Trans.Aerosp. Electron. Syst. 40(3), 851-861 (2004)

13. H He, P Stoica, J Li, in 2010 2nd International Workshop on Cognitive Information Processing (CIP). Waveform Design with Stopband and Correlation Constraints for Cognitive Radar (IEEE, Elba Island, 2010), pp. 344-349 
14. G Wang, Y Lu, Designing Single/Multiple Sparse Frequency Waveforms with Sidelobe Constraint. IET Radar Sonar Navig. 5(1), 32-38 (2011)

15. L Patton, CA Bryant, B Himed, in 2012 IEEE Radar Conference. Radar-Centric Design of Waveforms with Disjoint Spectral Support (IEEE, Atlanta, GE, 2012), pp. 1106-1110

16. N Li, G Cui, H Yang, L Kong, Q Liu, S lommelli, Adaptive Detection of Moving Target with MIMO Radar in Heterogeneous Environments Based on Rao and Wald Tests. Signal Process. 114(1), 198-208 (2015)

17. J Li, JR Guerci, L Xu, Signal Waveform's Optimal-under-Restriction Design for Active Sensing. IEEE Sig. Process. Lett. 13(9), 565-568 (2006)

18. A De Maio, S De Nicola, Y Huang, ZQ Luo, S Zhang, Design of Phase Codes for Radar Performance Optimization with a Similarity Constraint. IEEE Trans. Signal Process. 57(2), 610-621 (2009)

19. A Aubry, A De Maio, A Farina, M Wicks, Knowledge-Aided (Potentially Cognitive) Transmit Signal and Receive Filter Design in Signal-Dependent Clutter. IEEE Trans. Aerosp. Electron. Syst. 49(1), 93-117 (2013)

20. A Aubry, A De Maio, M Piezzo, A Farina, M Wicks, Cognitive Design of the Receive Filter and Transmitted Phase Code in Reverberating Environment. IET Radar Sonar Navig. 6(9), 822-833 (2012)

21. A Aubry, A De Maio, MM Naghsh, Optimizing Radar Waveform and Doppler Filter Bank via Generalized Fractional Programming. IEEE J Sel. Top. Signal Process. 9(8), 1387-1399 (2015)

22. A Turlapaty, Y Jin, in 2014 IEEE Radar Conference. A Joint Design of Transmit Waveforms for Radar and Communications Systems in Coexistence (Institute of Electrical and Electronics Engineers (IEEE), Cincinnati, 2014), pp. 315-319

23. A Aubry, A De Maio, M Piezzo, A Farina, Radar Waveform Design in a Spectrally Crowded Environment via Non-convex Quadratic Optimization. IEEE Trans. Aerosp. Electron. Syst. 50(2), 1138-1152 (2014)

24. A Aubry, AD Maio, Y Huang, M Piezzo, A Farina, A New Radar Waveform Design Algorithm with Improved Feasibility for Spectral Coexistence. IEEE Trans. Aerosp. Electron. Syst. 51(2), 1029-1038 (2015)

25. A Aubry, V Carotenuto, AD Maio, Forcing Multiple Spectral Compatibility Constraints in Radar Waveforms. IEEE Sig. Process. Lett. 23(4), 483-487 (2016)

26. AR Chiriyath, B Paul, GM Jacyna, DW Bliss, Inner Bounds on Performance of Radar and Communications Coexistence. IEEE Trans. Signal Process. 64(2), 464-474 (2016)

27. P Ge, G Cui, SM Karbasi, L Kong, J Yang, A Generalized Fitting Approach for Cognitive Unimodular Waveform Design. Signal Proc. 128(1), 360-368 (2016)

28. EA Lee, DG Messerschmitt, Digital Communication, 2nd edn, (Kluwer Academic, Boston, 1994)

29. B Sklar, Digital Communications:Fundamentals and Applications, 2nd edn. (Prentice Hall, Upper Saddle River, 2001)

30. H He, J Li, P Stoica, Waveform Design for Active Sensing Systems - A Computational Approach. (Cambridge Press, Wellesley, 2012)

31. L Han, K Wu, in Proceedings of the Asia-Pacific Microwave Conference 2011. Emerging Advances in Transceiver Technology Fusion of Wireless Communication and Radar Sensing Systems (IEEE, Melbourne, 2012), pp. 951-954

32. JG Metcalf, C Sahin, SD Blunt, M Rangaswamy, Analysis of Symbol-Design Strategies for Intrapulse Radar-Embedded Communications. IEEE Trans. Aerosp. Electron. Syst. 51(4), 2914-2931 (2015)

33. D Ciuonzo, A De Maio, M Piezzo, Intrapulse Radar-Embedded Communications via Multiobjective Optimization. IEEE Trans. Aerosp. Electron. Syst. 51(4), 2960-2974 (2015)

34. AR Chiriyath, B Paul, GM Jacyna, DW Bliss, Inner Bounds on Performance of Radar and Communications Coexistence. IEEE Trans. Signal Process. 64(2), 464-474 (2016)

35. GL Cui, XX Yu, V Carotenuto, LJ Kong, Space-Time Transmit Code and Receive Filter Design for Colocated MIMO Radar. IEEE Trans. Signal Process. 65(5), 1116-1129 (2017)

\section{Submit your manuscript to a SpringerOpen ${ }^{\circ}$ journal and benefit from:}

- Convenient online submission

- Rigorous peer review

- Immediate publication on acceptance

- Open access: articles freely available online

- High visibility within the field

- Retaining the copyright to your article

Submit your next manuscript at $\boldsymbol{s p r i n g e r o p e n . c o m ~}$ 